\title{
Distribuição dos casos de AIDS em mulheres no Rio de Janeiro, de 1982 a 1997: uma análise espacial
}

\author{
Distribution of AIDS cases in women \\ in Rio de Janeiro, Brazil, 1982-1997: \\ a spatial analysis
}

Jeane Tomazelli 1

Dina Czeresnia 2

Christovam Barcellos 3

\footnotetext{
1 Secretaria de Estado de Saúde do Rio de Janeiro. Rua México 128, 3o andar, Rio de Janeiro, $R J$ 20031-142, Brasil. jtomazelli@saude.rj.gov.br 2 Departamento de Epidemiologia e Métodos Quantitativos em Saúde, Escola Nacional de Saúde Pública, Fundação Oswaldo Cruz. Av. Leopoldo Bulhões 1480, Rio de Janeiro, $R J$ 21041-010, Brasil. dina@ensp.fiocruz.br 3 Departamento de Informações em Saúde, Centro de Informação Científica e Tecnológica, Fundação Oswaldo Cruz. Av. Brasil 4365, Rio de Janeiro, $R J$ 21045-900, Brasil. xris@cict.fiocruz.br
}

\begin{abstract}
The AIDS epidemic has spread and reached various population groups differently. The epidemic's dynamics have also differed according to the characteristics of different areas within cities, related to the principal modes of spread. This study analyzes the AIDS epidemic in women in the city of Rio de Janeiro using the space referential. The epidemic is on the rise among women, particularly in the Northern and Western Zones of the city. In this group it constitutes a "sub-epidemic", to the extent that it displays different characteristics in terms of clinical presentation, access to health services, and knowledge of risks. There was a high proportion of unknown transmission category among the women, thus revealing ignorance of their own risk situation. In addition, the high proportion of unknown level of schooling emphasizes the implications of the investigative system on quality of data recorded for women.
\end{abstract}

Key words HIV; Acquired Immunodeficiency Syndrome; Spatial Analysis

Resumo A epidemia de AIDS vem crescendo e atingindo de forma diferenciada os diversos grupos populacionais. Sua dinâmica dentro das cidades também vem mostrando-se distinta segundo as características de cada região. Diferenciais internos das cidades vêm sendo relacionados a modos principais de propagação. Este trabalho analisa a epidemia de AIDS em mulheres no Município do Rio de Janeiro, usando o referencial espacial. Observa-se o crescimento da epidemia da AIDS entre mulheres atingindo mais intensamente regiões das Zonas Norte e Oeste. A epidemia neste grupo compõe uma "subepidemia" na medida em que possui características clínicas, de acesso ao serviço e de conhecimento de riscos diferentes. Destaca-se a elevada proporção na categoria de transmissão ignorada dentre as mulheres, evidenciando um desconhecimento de sua situação de risco. Alta proporção de escolaridade ignorada, reforça a implicação do sistema de investigação sobre a qualidade da informação em mulheres.

Palavras-chave HIV; Síndrome de Imunodeficiência Adquirida; Análise Espacial 


\section{Introdução}

A epidemia de AIDS vem mostrando um perfil dinâmico desde seu surgimento. A infecção pelo HIV, inicialmente vinculada a homossexuais masculinos, disseminou-se atingindo pessoas de diferentes idades, sexos, opções sexuais, raças, comunidades e países.

No Brasil, a AIDS também se manifestou de forma distinta em segmentos populacionais (Szwarcwald \& Bastos, 2000). A compreensão e análise da transmissão da doença necessitaram progressivamente do reconhecimento de interações sociais cada vez mais complexas (Bastos et al., 1995). Nesse processo, destaca-se o crescimento da AIDS entre as mulheres.

O conhecimento acerca da AIDS em mulheres e as campanhas preventivas específicas para elas, desenvolveram-se preferencialmente com base nas mulheres em fase de reprodução. A soropositividade é detectada, na maioria das vezes, na gravidez ou após a morte ou adoecimento de filhos ou companheiros. As mulheres geralmente são informadas de seu diagnóstico, freqüentemente tardio, e recebem tratamento quando adoecem (Barbosa, 1996; Diniz \& Villela, 1999; Vermelho, 1999).

$\mathrm{O}$ aumento do número de casos de AIDS em mulheres já podia ser observado desde o ano de 1986. Em 1989, o crescimento da AIDS feminina foi paralelo ao aumento do número de casos em usuários de drogas injetáveis, ocasionando uma tendência de identificar as mulheres infectadas com este segmento. Atualmente, a transmissão sexual apresenta-se como o principal modo de transmissão da AIDS (Castilho \& Szwarcwald, 1988; MS, 2000).

Junto a esta nova dinâmica, destaca-se a subnotificação de casos femininos, devido à captação tardia da soropositividade. Além disso, a operacionalização das estratégias preventivas baseadas no conceito de grupo de risco, exclui as mulheres que destoam desse estereótipo fazendo com que algumas suspeitas sejam descartadas (Diniz \& Villela, 1999).

O processo de feminização da epidemia é concomitante ao de pauperização, ou seja, o aumento de casos em mulheres acompanha a expansão da epidemia para grupos sociais mais pobres (Szwarcwald \& Bastos, 2000). Todavia, a magnitude do processo de pauperização da infecção pode estar superestimada. As pessoas que acorrem mais aos serviços públicos são pertencentes a grupos mais pobres, com acesso diferenciado aos anti-retrovirais. Esse grupamento comparado com outros segmentos sociais pode apresentar tempo menor entre a infecção e o adoecimento (Szwarcwald \& Bastos,
2000). Destaca-se ainda a captação diferenciada de casos pela rede de saúde, em função da estruturação do próprio serviço, produzindo variações de forma enviesada na notificação por fatores sociais e econômicos (Parker, 1994; Parker \& Camargo, 2000).

De qualquer forma, a análise de dados de notificação tem demonstrado que a epidemia de AIDS no Brasil está crescendo entre as mulheres, disseminando-se para segmentos de menor escolaridade do que os registrados no início da epidemia. Os municípios mais pobres têm apresentado maior incremento das taxas de incidência ao longo da última década (Szwarcwald \& Bastos, 2000).

A forma de se construir o conhecimento acerca da AIDS em mulheres, pode estar subestimando nuanças importantes devido ao desconhecimento da história de infecção, visto que muitas dessas mulheres não suspeitavam de sua condição de infecção pelo HIV. A omissão de informações é outro aspecto relevante devido ao fato da epidemia AIDS envolver a intimidade das pessoas. Pode ocorrer também superposição de categorias de transmissão. Somase a tudo isso os limites dos sistemas de informação, que muitas vezes operacionalizados de forma insatisfatória causam distorções.

Evidencia-se a necessidade de discriminar melhor quem são essas mulheres em suas características culturais, sociais, econômicas, comportamentais e intersubjetivas no sentido de buscar apreender como estas dimensões se articulam e propiciam o crescimento da epidemia entre as mulheres.

A dinâmica da epidemia tem apontado para o seu caráter global e simultaneamente local, referindo-se a comunidades específicas. A AIDS é uma epidemia mundial que assume características peculiares aos diversos grupos culturais na qual emerge (Gould, 1993). Deve, portanto, ser compreendida como produto de diferentes culturas, dos diversos comportamentos e do agente infeccioso (Bastos \& Coutinho, 1999). Os processos globais não atingem diretamente os indivíduos, mas são mediados por fatores que ocorrem em "meso-escalas", relativas às comunidades, onde pessoas vivem $\mathrm{e}$ moldam seu comportamento (Wallace \& Wallace, 1997).

As formas de exposição e adoecimento por AIDS são já bem conhecidas. Alguns fatores de risco, no entanto, envolvem componentes de escala maior que o indivíduo, e só podem ser compreendidos por meio de uma visão estrutural e social da epidemia (Parker et al., 2000). Uma das maneiras de se alargar a visão sobre o problema é a sistematização de dados em agre- 
gados que melhor retratem as profundas desigualdades sociais da epidemia no mundo. A proposta deste trabalho, de produzir um diagnóstico da AIDS com foco no território e na população, é uma contribuição para o entendimento do contexto em que a epidemia de AIDS vem se difundindo no meio urbano. Dessa maneira, permite caracterizar a situação de saúde, bem como estabelecer estratégias para a prevenção, voltadas para grupos vulneráveis dentro de um contexto global.

Este artigo se propõe a analisar a dinâmica da epidemia de AIDS em mulheres no Município do Rio de Janeiro, entre 1982 e 1987, utilizando uma abordagem espacial.

Estudos epidemiológicos que analisam a epidemia segundo a organização do espaço, compreendem a transmissão da AIDS por meio do conceito de interação. Esse conceito permite estudar a difusão das epidemias de forma mais complexa que o conceito de risco. Diferentes indivíduos e grupos sociais participam de forma diferenciada de redes de interação social que determinam dinâmicas peculiares e configurações espaciais específicas (Barcellos \& Bastos, 1996).

Trabalhos como os de Grangeiro (1994) e Cruz (1999), revelam um comportamento epidêmico da AIDS vinculado a condições de vida das regiões. Assim, áreas com piores condições de vida apresentaram aumento no número de casos, mostrando um deslocamento espacial da epidemia. O tamanho da região e a sua inserção econômica e social também são apontados como aspectos importantes na difusão da epidemia (Barcellos \& Bastos, 1996).

Estabelecendo-se as características da população e a sua localização, é possível mediante a visualização das informações nos mapas, criar hipóteses e questionamentos sobre agravos à saúde, reconhecendo-se áreas mais vulneráveis (Santos et al., 2000).

\section{Metodologia}

Foi realizado um estudo ecológico para analisar a distribuição espacial da incidência dos casos notificados de AIDS em mulheres, de 15 a 59 anos de idade, no Município do Rio de Janeiro, ocorridos no período entre 1982 e 1997. Utilizou-se análise espacial como técnica de tratamento de dados e o bairro como unidade de análise.

Análise espacial é um conjunto de técnicas de tratamento de dados, envolve a manipulação de dados com base na integração de informações com referências espaciais, com o obje- tivo de compreender as relações entre as variáveis espacialmente localizadas.

Foram utilizados como fontes de dados o Sistema de Informação de Agravos de Notificação (SINAN-AIDS) e os censos demográficos de 1991 e 2000. Os dados foram disponibilizados pelo Programa de DST/AIDS da Secretaria Municipal de Saúde, com todos os casos de AIDS notificados no Município do Rio de Janeiro, relativos ao período de 1982 a 1999. Devido ao atraso das notificações nesse sistema (Barbosa \& Struchiner, 1997), foram considerados apenas os casos notificados até 1997. Os casos de AIDS notificados foram divididos segundo períodos, de acordo com o sexo e a categoria de exposição ao HIV.

As variáveis abordadas neste estudo foram: bairro (unidade do município onde reside o caso notificado), escolaridade (grau de instrução: analfabeto, 1a a 4a série, 5a a $8 \underline{a}$ série, $2 \underline{o}$ grau, superior e ignorado), idade, ano epidemiológico (classificado segundo períodos de 1982-1988, 1989-1993 e 1994-1997), sexo, faixa etária (classificadas por grupos 15-24 anos, 25-34, 35-44 e 45-59 anos) e período (intervalo de tempo comum a um grupo e categoria de transmissão).

A delimitação dos períodos foi realizada após análise exploratória da freqüência da epidemia nas mulheres. O início e fim de cada período foram definidos segundo mudanças mais marcantes no incremento da notificação de casos em mulheres.

Foram criados os seguintes indicadores:

- Taxa de incidência: casos de AIDS ocorridos entre 15-59 anos no período, por sexo, ocorridos nos bairros do Município do Rio de Janeiro, em relação à população estimada para o período, por sexo nos bairros.

- Percentual de casos de AIDS com categoria de exposição ignorada: casos de AIDS com categoria de exposição ignorada ocorridos entre 15-59 anos, no período 1982-1997, nos bairros do Município do Rio de Janeiro, por sexo, em relação ao total de casos ocorridos no mesmo período, por sexo nos bairros.

- Razão de sexo: todos os casos de AIDS em homens ocorridos entre 15-59 anos nos bairros do Município do Rio de Janeiro, por período, em relação a todos os casos de AIDS em mulheres 15-59 anos, por período nos bairros.

- Percentual de escolaridade até 4a série: número de chefes de família com escolaridade até 4a série, por bairro, em relação ao número total de chefes de família, por bairro, utilizando o censo de 1991.

- Percentual de mulheres com AIDS com escolaridade até $4 \underline{a}$ série: casos de mulheres com AIDS com escolaridade até 4 a série, por bairro, 
em relação ao total de mulheres com AIDS por bairro.

- Percentual de homens com AIDS com escolaridade até 4 a série: casos de homens com AIDS com escolaridade até 4a série, por bairro, em relação ao total de homens com AIDS por bairro.

- Proporção de chefes de domicílio com renda superior a 10 salários mínimos: números de chefes de domicílios com renda superior a 10 salários mínimos em relação ao total de chefes de domicílios

Para análise dos dados foram utilizados os programas SPSS for Windows, Excel e o Map Info.

\section{Resultados}

No primeiro período (1982-1988), o número de casos em mulheres, apesar de crescente, é ainda incipiente. De 1988 a 1993, o número tornase mais expressivo e, entre 1994 a 1997, acentua-se a tendência de crescimento dos casos femininos. A análise da distribuição dos casos em mulheres, notificados por faixa etária e ano epidemiológico, mostrou uma concentração de casos nas faixas 25-34 e 35-44 anos.

O mapa de incidência dos casos notificados de AIDS em mulheres segundo os períodos previamente delimitados (Figura 1), mostrou o aumento da incidência e a sua dinâmica pelo município: no primeiro período, 1982-1988, evidenciou-se maiores taxas em bairros das regiões centrais da cidade, provável "epicentro" da epidemia (Wallace \& Wallace, 1995). Outros bairros apresentam baixas taxas de incidência mostrando um aspecto difuso de casos. No segundo e terceiro períodos, 1989-1993 e 19941997, pode-se observar o aumento da incidência e a disseminação da epidemia por todo município, com uma tendência de crescimento de casos notificados no sentido Leste-Oeste. Estes bairros vão tendendo a apresentar uma incidência cada vez mais acentuada. Os bairros da Zona Oeste, assim como da Zona Norte, apresentam condições materiais de vida mais precária (Cruz, 1999), como pode ser evidenciado pela comparação com o mapa de renda de chefes de família (Figura 2). No último período, é notável o aumento do número de casos ao longo da Baía de Guanabara, na chamada Zona da Leopoldina, onde se concentra a maior parte das favelas do Rio de Janeiro. O crescimento da epidemia nestas regiões pode estar também vinculado ao processo de pauperização, como refere a literatura. Todavia, existem também bairros na chamada Zona Sul, como Botafogo, com aumento da incidência nos dois últimos períodos.

A Figura 3 estabelece a razão de casos de AIDS entre homens e mulheres $(\mathrm{H} / \mathrm{M})$ nos três períodos. As áreas em branco no mapa representam bairros que não tiveram registro de casos masculinos ou femininos em cada período.

Pode-se observar a redução da razão $\mathrm{H} / \mathrm{M}$ ao longo dos três períodos. No início da epidemia, a maior parte dos bairros apresentava razão acima de quatro. Bairros da Zona Sul com grande incidência de casos apresentavam razão H/M superior a 10 . No último período observa-se a tendência de diminuição da razão H/M: os bairros das Zonas Norte e Oeste, com poucas exceções, apresentaram razão menor que quatro. Bairros da Zona Sul e o Centro permaneceram com razões de casos por sexo alta - entre quatro e dez - corroborando o predomínio de casos masculinos nestes bairros ao longo de todos os períodos. Nas Zonas Centro e Sul, o perfil da epidemia ainda está mais próximo ao da década de 80.

Esses dados reforçam a hipótese de que o processo de feminização acompanha o de pauperização. Por outro lado, mostra uma estranha separação entre epidemias masculina, atingindo a Zona Sul da cidade, e feminina, nas Zonas Norte e Oeste. Essa tendência pode ser conseqüência da predominância de formas de exposição diferenciadas que se refletem na divisão de bairros da cidade.

Isso se confirma com a análise da informação de escolaridade dos casos. Como demonstra a Tabela 1, a escolaridade de todos os casos tendeu a diminuir no decorrer dos três períodos. Porém, a escolaridade dos casos masculinos é proporcionalmente mais alta que a dos femininos: $15,2 \%$ de casos masculinos têm escolaridade acima de segundo grau, enquanto somente 5,9\% dos femininos têm esse nível de escolaridade. A qualidade da informação acerca da escolaridade é apenas um pouco melhor para os homens do que para as mulheres: $23,5 \%$ e $26,5 \%$ de casos com informação ignorada, respectivamente, no último período. Nota-se a tendência de melhoria do preenchimento desta informação no decorrer dos três períodos.

A Figura 4 mostra a distribuição e o porcentual do nível de escolaridade até 4 a série na população em geral (segundo dados do censo), homens e mulheres com AIDS.

Assim como o padrão de renda, observa-se que na população em geral (dados do Censo Demográfico de 1991 - IBGE, 1991), as melhores condições de escolaridade concentram-se na Zona Sul da cidade, sendo menores na Zona Oeste. O Centro e os bairros da Zona Norte 
Taxa de incidência dos casos notificados de AIDS em mulheres ao longo dos períodos.

Município do Rio de Janeiro, Brasil.

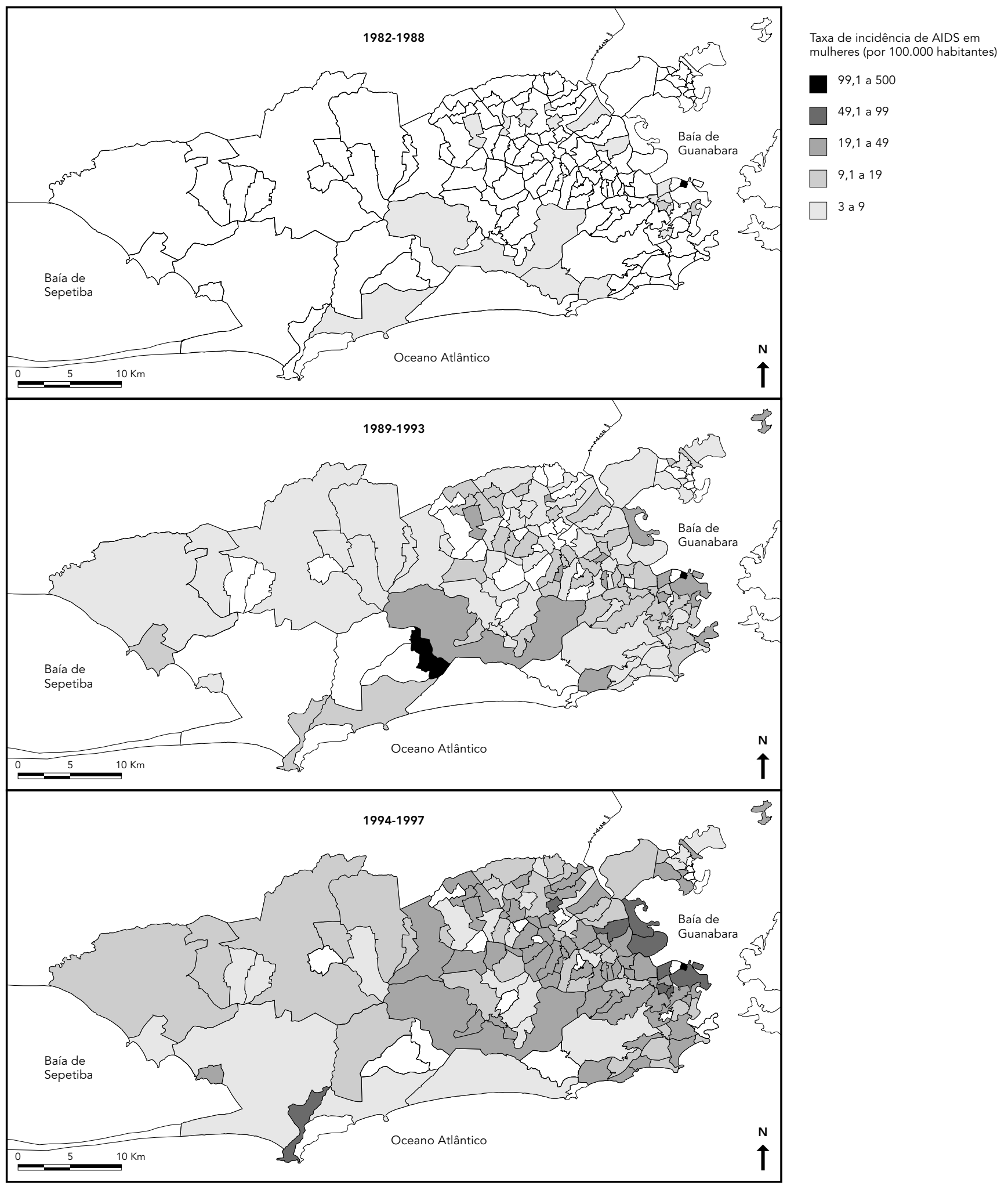




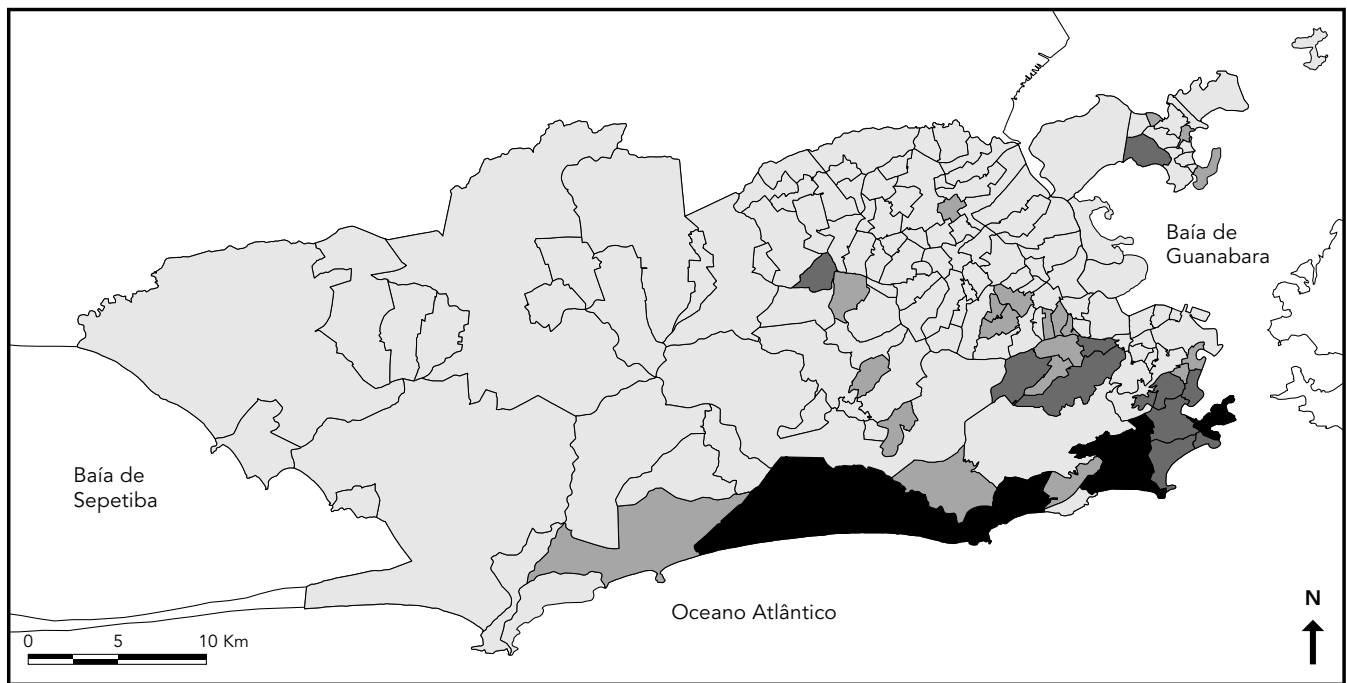

Proporção de chefes com renda superior a 10 salários mínimos
51,6 e mais
34,4 a 51,5
17,2 a 34,3
0 a 17,1

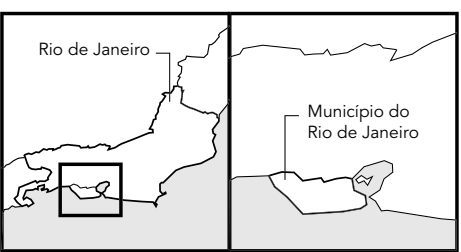

apresentam níveis intermediários de escolaridade.

O nível de escolaridade dos casos notificados de AIDS em mulheres apresentou-se muito baixo, com um percentual de escolaridade até a 4 a série acima de $60 \%$ em vários bairros. Destaca-se um núcleo formado pelos bairros Manguinhos, Bonsucesso, Higienópolis e Jacaré e alguns da Zona Norte com porcentual superior a $80 \%$.

Pode-se observar que as pessoas com AIDS possuem, proporcionalmente, uma escolaridade menor do que a população em geral. Além disso, a escolaridade dos casos de AIDS em mulheres é, geralmente, menor do que a dos casos masculinos.

O preenchimento da informação sobre o modo de transmissão da AIDS entre os casos notificados, apresenta característica bem diferente da informação sobre escolaridade e merece ser analisada em destaque. Certamente, por dizer respeito a uma dimensão mais complexa da realidade dos sujeitos, portanto, mais difícil de ser relatada, o preenchimento do modo de transmissão nas fichas de notificação é de pior qualidade. Como se pode observar na Tabela 2, a categoria de exposição, além de ser uma variável que apresenta proporcionalmente maior quantidade de informação ignorada do que a de escolaridade, tendeu, ao contrário desta, a piorar a qualidade do preenchimento no decorrer dos três períodos, tanto para homens como para mulheres. A dimensão da informação ignorada a respeito do modo de transmissão, também ao contrário da informação sobre escolaridade, é expressivamente maior entre as mulheres do que entre os homens: $49,8 \%$ e $36,8 \%$, respectivamente, no último período.

A tendência de piora da qualidade da informação sobre categoria de exposição no decorrer do tempo, pode sugerir a hipótese de que esta qualidade piora na medida em que a AIDS atinge proporcionalmente grupos mais empobrecidos da população do município.

A Figura 5 mostra a distribuição do porcentual de casos de AIDS nos bairros, com catego- 
Figura 3

Razão de casos de AIDS entre homens e mulheres. Município do Rio de Janeiro, Brasil.

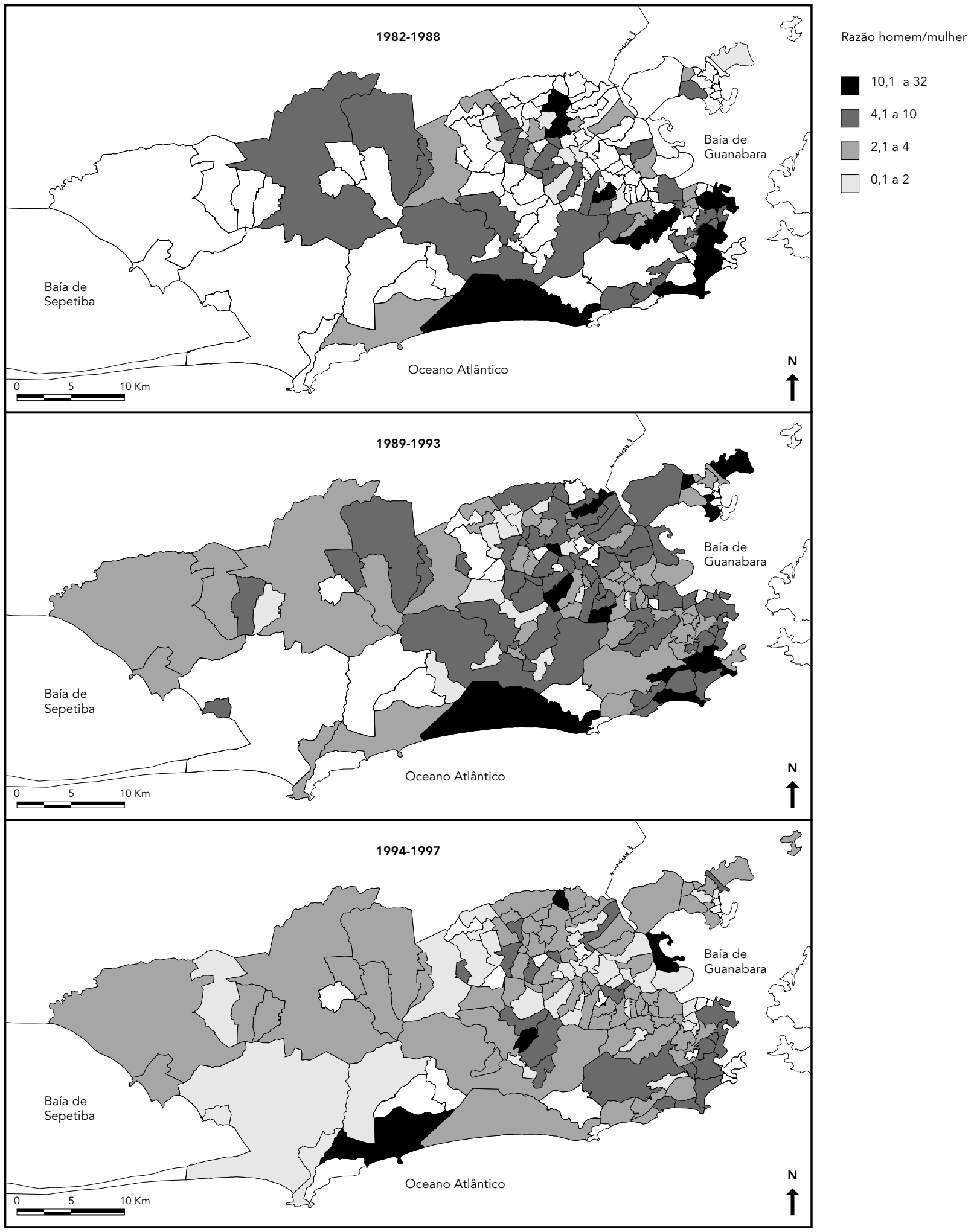


Tabela 1

Distribuição dos casos de AIDS em mulheres e homens de 15-59 anos, segundo período e escolaridade.

\begin{tabular}{|c|c|c|c|c|c|c|}
\hline \multirow[t]{2}{*}{ Escolaridade } & \multicolumn{3}{|c|}{ Mulher } & \multicolumn{3}{|c|}{ Homem } \\
\hline & 1982-1988 & 1989-1993 & 1994-1997 & 1982-1988 & 1989-1993 & 1994-1997 \\
\hline Analfabeto & $4(2,3 \%)$ & $34(3,4 \%)$ & $112(5,4 \%)$ & $12(0,8 \%)$ & $69(1,3 \%)$ & $150(2,5 \%)$ \\
\hline 1ạ-4a série & $54(30,9 \%)$ & $355(35,5 \%)$ & 958 (46,1\%) & 313 (21,4\%) & $1.244(23,7 \%)$ & $2.147(35,5 \%)$ \\
\hline 5a-8a série & $22(12,6 \%)$ & $154(15,4 \%)$ & $336(16,2 \%)$ & $240(16,4 \%)$ & $991(18,9 \%)$ & $1.412(23,3 \%)$ \\
\hline 2o grau & $23(13,1 \%)$ & $113(11,3 \%)$ & $123(5,9 \%)$ & $306(21,0 \%)$ & $1.034(19,7 \%)$ & $923(15,2 \%)$ \\
\hline Superior & $7(4,0 \%)$ & $32(3,2 \%)$ & - & $130(8,9 \%)$ & $532(10,1 \%)$ & $1(0,1 \%)$ \\
\hline Ignorado & $65(37,1 \%)$ & $311(31,1 \%)$ & $550(26,5 \%)$ & $459(31,4 \%)$ & $1.374(26,2 \%)$ & $1.423(23,5 \%)$ \\
\hline Total & $175(100,0 \%)$ & 999 (100,0\%) & $2.079(100,0 \%)$ & $1.460(100,0 \%)$ & $5.244(100,0 \%)$ & $6.056(100,0 \%)$ \\
\hline
\end{tabular}

Fonte: Sistema Informação de Agravos de Notificação.

ria de exposição ignorada, segundo o sexo, de 1982-1997. A proporção de mulheres com categoria de exposição ignorada é, em geral, maior do que a dos homens. Esta diferença de porcentual é maior nas áreas Norte e Oeste do município. Casos de homens com categoria de exposição ignorada apresentaram-se menos freqüentes e com um caráter mais difuso.

Vargem Grande e a Vila Militar foram bairros que apresentaram elevado percentual de categoria de transmissão ignorada, tanto para homens como para mulheres. Contudo, foram bairros com poucos casos notificados.

\section{Discussão}

O trabalho confirma que a AIDS está se disseminando em direção a bairros mais empobrecidos do município, localizados em sua maioria nas Zonas Norte e Oeste. O crescimento da epidemia nos bairros mais pobres é mais intenso entre as mulheres, especialmente as de baixa escolaridade e renda.

A distribuição espacial da AIDS no Município do Rio de Janeiro, sugere a existência de um complexo mecanismo de difusão do HIV entre áreas e grupos sociais. Três tipos de difusão espacial de epidemias foram destacados por Wallace \& Wallace (1997). A difusão hierárquica de agentes infecciosos envolve a transmissão "em cascatas" de locais dominantes para outros, de posição social inferior. Outra possibilidade de difusão é por meio de contiguidade, permitindo o contágio entre áreas adjacentes devido à freqüência de interações entre vizinhos. Finalmente, a difusão por redes permite a transmissão dentro de comunidades, entre pessoas participantes de redes sociais. Neste trabalho é evidenciada uma separação entre diferentes epidemias na cidade, uma predominantemente masculina, situada na zona sul, e outra, com equilíbrio entre casos femininos e masculinos, afetando bairros e pessoas de baixa renda. Esse padrão sócioespacial é raramente observado nos estudos nacionais, tendo os municípios ou estados como unidade de análise (Gould, 1993; Szwarcwald, et al., 2000a), o que pode evidenciar a existência de um fenômeno específico da escala intra-urbana. Os mecanismos de difusão por redes ou contiguidade reproduzem, em geral, a estrutura sócioespacial da cidade, já que pressupõem a interação entre semelhantes. No caso em estudo, ao contrário, o processo de difusão do HIV vem alterando o perfil da epidemia nos locais onde o vírus é introduzido. Devido a limitações metodológicas, não se pode afirmar se a pauperização da epidemia é causa ou conseqüência da sua difusão. A predominância de grupos vulneráveis, marcadamente mulheres de baixa renda, nas Zonas Norte e Oeste da cidade, pode estar facilitando o rápido espalhamento do HIV nessas áreas. Por outro lado, essas áreas se caracterizam pela introdução recente do HIV e no estágio atual da epidemia no Brasil, as mulheres de baixa renda constituem grupos mais vulneráveis na hierarquia da difusão. Esses resultados revelam a interação entre os grupos locais suscetíveis, configurando redes sociais que ultrapassam os limites dos bairros de residência.

Assim, a difusão do HIV nas cidades tem sido promovida pela proximidade de comunidades periféricas com áreas e grupos de maior prevalência, e pela desestruturação social interna dessas comunidades (Wallace et al., 1997). Nesse sentido, o autor tem destacado a "desertificação urbana" e o abandono por parte dos serviços públicos como determinantes não só do recrudescimento da epidemia de AIDS, mas 
Percentual de pessoas com escolaridade até a 4ạ série. Município do Rio de Janeiro, Brasil.

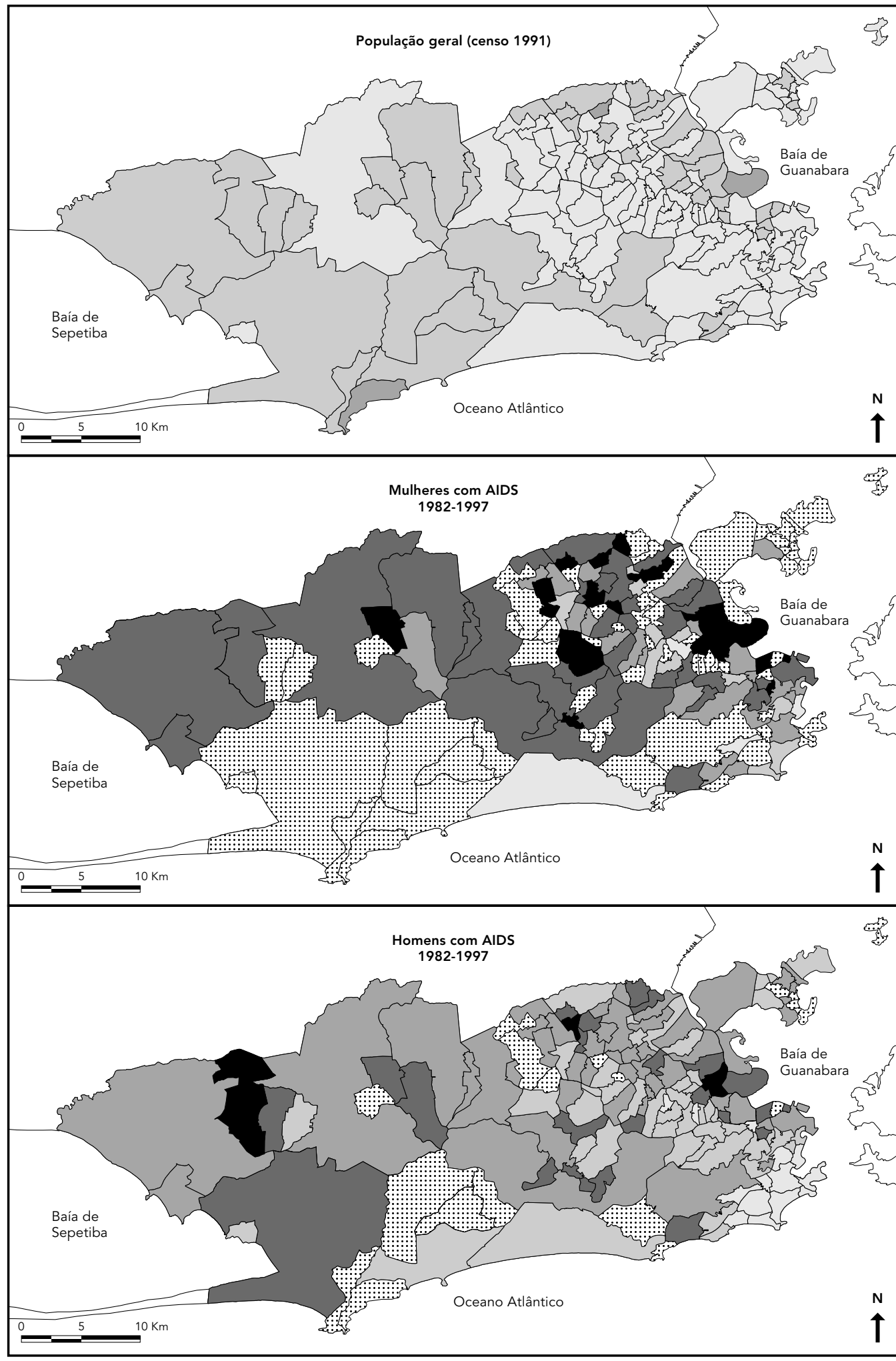

Percentual de pessoas com escolaridade até a 4 a série

80,1 a 100

60,1 a 80

40,1 a 60

20,1 a 40

0 a 20

Bairros excluídos

(>8 casos) 
Tabela 2

Categoria de transmissão ignorada por sexo, segundo o período.

\begin{tabular}{|c|c|c|c|c|c|c|}
\hline \multirow{2}{*}{$\begin{array}{l}\text { Sexo } \\
\text { Feminino }\end{array}$} & \multicolumn{6}{|c|}{ Período } \\
\hline & \multicolumn{2}{|c|}{ 1982-1988 } & \multicolumn{2}{|c|}{ 1989-1993 } & \multicolumn{2}{|c|}{ 1994-1997 } \\
\hline Categoria ignorada & 35 & $20,0 \%$ & 314 & $31,4 \%$ & 1.035 & $49,8 \%$ \\
\hline Outras categorias & 140 & $80,0 \%$ & 685 & $68,6 \%$ & 1.044 & $50,2 \%$ \\
\hline Total & 175 & $100,0 \%$ & 999 & $100,0 \%$ & 2.079 & $100,0 \%$ \\
\hline \multicolumn{7}{|l|}{ Masculino } \\
\hline Categoria ignorada & 275 & $18,8 \%$ & 1.294 & $24,7 \%$ & 2.227 & $36,8 \%$ \\
\hline Outras categorias & 1.185 & $81,2 \%$ & 3.950 & $75,3 \%$ & 3.829 & $63,2 \%$ \\
\hline Total & 1.460 & $100,0 \%$ & 5.244 & $100,0 \%$ & 6.056 & $100,0 \%$ \\
\hline
\end{tabular}

Fonte: Sistema Informação de Agravos de Notificação.

também da violência, tuberculose e outros indicadores de qualidade de vida. Na mesma Zona Norte do Rio de Janeiro, foi observada uma redução significativa da expectativa de vida em áreas pobres com grande concentração de favelas (Szwarcwald et al., 2000b).

Pode-se observar que a escolaridade dos casos notificados de AIDS foi menor do que a da população em geral. O nível de escolaridade dos casos notificados femininos é menor do que o dos homens. A escolaridade é um indicador utilizado na análise de dados epidemiológicos para aferir a condição sócio-econômica da população, onde baixos níveis educacionais estão relacionados com estratos sociais mais pobres. Contudo, a avaliação da dinâmica da transmissão da AIDS em indivíduos com baixa escolaridade pode estar superestimada. Os casos de soropositividade à AIDS que adoecem são notificados logo que iniciam o tratamento. Assim sendo, o indicador estará fornecendo informações sobre a escolaridade daqueles que evoluem mais rapidamente para doença, mascarando a informação sobre a real dinâmica de transmissão da epidemia (Szwarcwald \& Bastos, 2000).

A maior velocidade de adoecimento pode estar acontecendo com mais freqüência nas mulheres, pois estas tendem a apresentar um diagnóstico de infecção pelo HIV mais tardio que o dos homens (Diniz \& Villela, 1999; Vermelho, 1999). O diagnóstico ocorrendo num processo mais avançado da infecção conduziria também a uma evolução mais rápida para a AIDS.

A qualidade da informação é um indicador da qualidade de assistência e indiretamente da condição sócio-econômica dos casos. A desin- formação sobre a escolaridade é proporcionalmente mais alta entre as mulheres do que entre os homens. Porém, o que chama mais a atenção é a diferença proporcional de informação não preenchida, entre homens e mulheres, no que diz respeito ao modo de transmissão. Essa desinformação vem crescendo relativamente ao longo dos períodos estudados, tanto para homens como para mulheres, mas, no caso das mulheres é expressivamente maior, apresentando-se espacialmente mais intensa nas Zonas Norte e Oeste.

Se o diagnóstico da soropositividade é mais tardio nas mulheres (Diniz \& Villela 1999; Vermelho, 1999), a defasagem de tempo entre a exposição e a notificação pode prejudicar a qualidade da informação sobre a forma de infecção, o que pode ser agravado em áreas pobres da cidade, sem serviços de saúde ou acesso à informação.

Sem dúvida, o processo de feminização está vinculado ao de pauperização da AIDS. Mas o maior diferencial da desinformação sobre a forma de infecção nas mulheres, além da maior proporção de casos femininos oriundos dos grupos mais pobres, e do diagnóstico tardio, provavelmente está relacionado a aspectos culturais de gênero. Para além da pior qualidade da assistência, em que medida as mulheres, em maior proporção, desconhecem, elas próprias, a forma como se contaminaram?

\section{Conclusão}

A adoção de bairro como unidade de análise, ressaltou os diferenciais internos da cidade, evidenciando processos sociais e epidemiológicos 
Figura 5

Percentual de casos de AIDS em homens e mulheres com categoria de exposição ignorada.

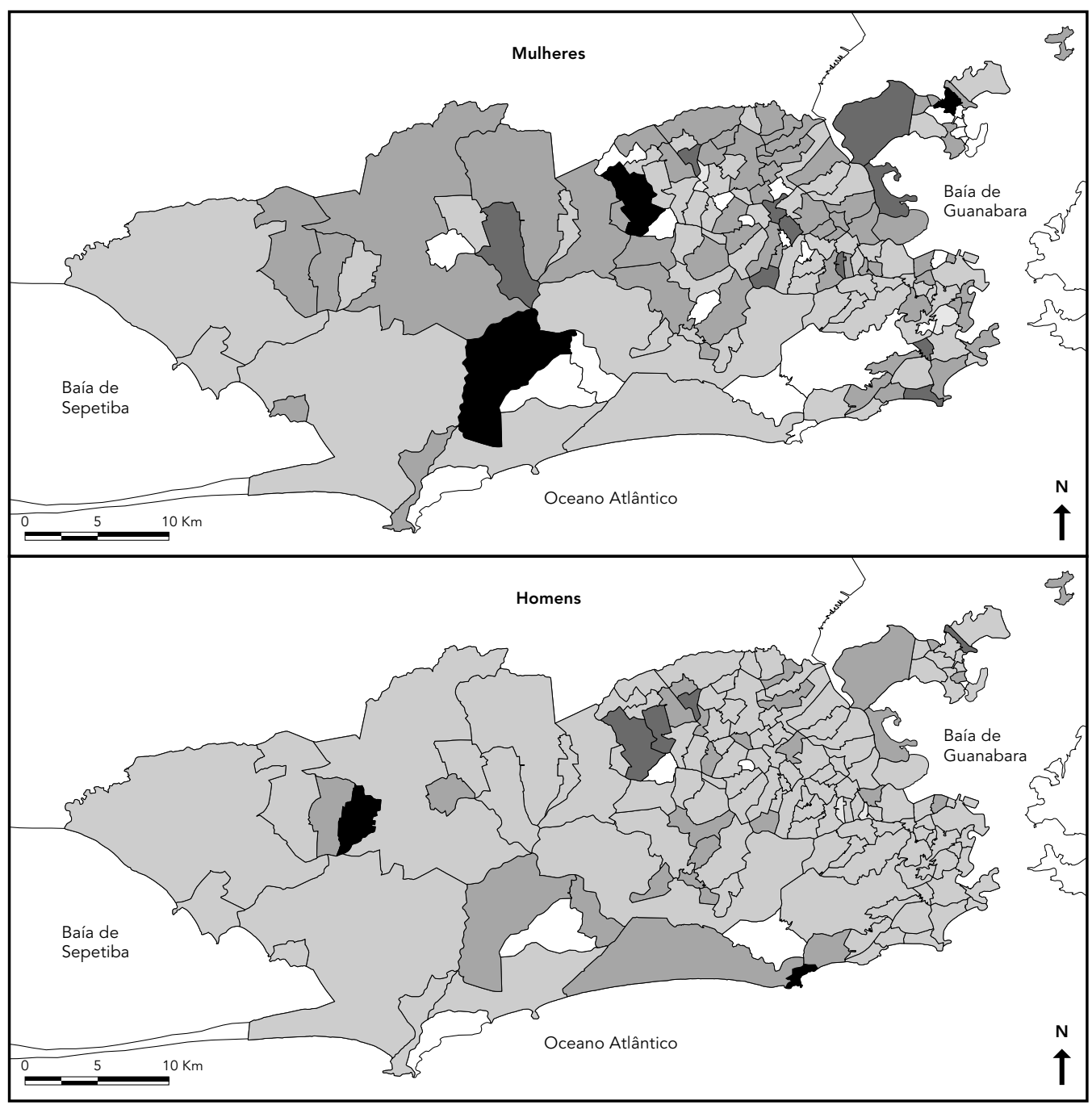

Percentual de ignorados

71 a 100
$\square \quad 51$ a 70
$\square \quad 31$ a 50
$\square \quad 11$ a 30
$\square \quad 5$ a 10

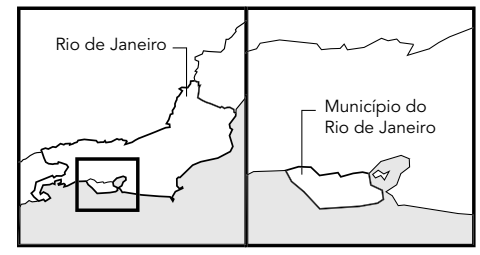


que ocorrem no seu interior. Outro aspecto importante que emergiu dessa escala foi o reflexo das desigualdades sociais sobre o acesso aos serviços, infra-estrutura urbana, perfis de renda. O reconhecimento desses contrastes intraurbanos permitiu que se ressaltasse o perfil social do município e seus possíveis reflexos sobre as condições de saúde da população.

Destaca-se que os mapas foram produzidos com base no local de residência dos casos e não necessariamente representam o local onde ocorreu a infecção. Apesar disso, as informações observadas espacialmente foram consideradas importantes na medida em que traduziram, de qualquer forma, informações sobre o perfil dos casos notificados residentes na unidade de análise.

A epidemia de AIDS adquire um perfil onde segmentos cada vez mais jovens são atingidos pela doença. A notificação dos casos na faixa etária anterior indica uma contaminação em faixas menores, englobando populações de adolescentes. Faz-se necessário desenvolver estratégias de prevenção mais eficazes para este grupamento populacional.

O estudo mostrou que a difusão da AIDS apresenta uma realidade distinta entre homens e mulheres. A tendência de crescimento da epidemia em mulheres está ocorrendo predominantemente nos bairros mais pobres e a qualidade da informação dos casos femininos é pior. Ainda que se constate o processo de feminização, os homens continuam sendo, em termos absolutos, o segmento predominante e provavelmente o "pólo difusor" da epidemia. Observou-se que as mulheres apresentaram desvantagens na qualidade dos dados registrados. Além do diferencial sócio-econômico, outros aspectos devem estar influindo na pior qualidade de informação dos casos femininos.
Valendo-se dos resultados da análise espacial realizada neste trabalho, pode-se esboçar um modelo do processo de difusão da AIDS para a cidade do Rio de Janeiro, que pode estar se repetindo em outras metrópoles do país. A introdução do HIV na cidade, como em quase todo o Brasil, foi caracterizada pela grande proporção de casos masculinos, de alta escolaridade, com transmissão homossexual do vírus. $\mathrm{Na}$ última década, esse perfil foi profundamente alterado com o aumento da importância da transmissão heterossexual com a diminuição da razão de casos entre homens e mulheres. No entanto, esse processo não é homogêneo no interior das cidades. No Rio de Janeiro, as áreas central e sul permanecem com perfis da epidemia semelhantes aos da década de 80, enquanto nas áreas mais pobres, o HIV se difunde preferencialmente entre mulheres de baixa renda e pouca escolaridade. As redes de interação social atuantes no meio urbano devem ser investigadas para se buscar explicações para esse processo. Pode-se levantar a hipótese de que mulheres residentes nessas áreas participem de interações com grupos sociais com alta prevalência de HIV/AIDS, fora de sua área de residência.

A análise espacial permite um nível de investigação até determinado limite, apontando para a necessidade de estudos com outras abordagens. Os resultados deste trabalho apontam a necessidade de estudos qualitativos, onde a questão de gênero pode explicar dimensões importantes da epidemia da AIDS em mulheres. Cabe ressaltar a necessidade de se investigar com mais profundidade os condicionantes da maior proporção de desinformação acerca do modo de transmissão nas mulheres. 


\section{Referências}

BARBOSA, M. T. S. \& STRUCHINER, C. J., 1997. Estimativas do número de casos de AIDS: Comparação de métodos que corrigem o atraso de notificação. In: Simpósio Satélite - A Epidemia da AIDS no Brasil: Situação e Tendências (Ministério da Saúde, org.), pp. 15-26, Brasília: Ministério da Saúde.

BARBOSA, R. H., 1996. Feminismo e AIDS. In: Quebrando o Silêncio Mulheres e AIDS no Brasil (R. Parker \& J. Galvão, org.), pp. 153-168, Rio de Janeiro: Relume Dumará/Associação Interdisciplinar de AIDS/Instituto de Medicina Social, Universidade do Estado do Rio de Janeiro.

BARCELLOS, C. \& BASTOS, F. I., 1996. Redes sociais e difusão da AIDS no Brasil. Boletín de la Oficina Sanitaria Panamericana, 121:11-24.

BASTOS, F. I. \& BARCELLOS, C., 1995. Geografia social da AIDS no Brasil. Revista de Saúde Pública, 29:52-62.

BASTOS, F. I. \& COUTINHO, K., 1999. Tão longe, tão perto... As pesquisas sobre HIV/AIDS no Brasil. In: Saúde, Desenvolvimento e Política - Resposta Frente à AIDS no Brasil (R. Parker, J. Galvão \& M. Bessa, org.), pp. 339-395, Rio de Janeiro: Editora 34.

BASTOS, F. I.; TELLES, P. R.; CASTILHO, E. \& BARCELLOS, C., 1995. A epidemia de AIDS no Brasil. In: Os Muitos Brasis - Saúde e População na Década de 80 (M. C. Minayo, org.), pp. 245-268, São Paulo: Editora Hucitec/Rio de Janeiro: ABRASCO.

CASTILHO, E. \& SZWARCWALD, C., 1998. Mais uma pedra no meio do caminho dos jovens brasileiros: A AIDS. In: Jovens Acontecendo na Trilha das Políticas Públicas (Comissão Nacional de População e Desenvolvimento - CNPD, org.), pp. 197-207, Brasília: CNPD.

CRUZ, M. M., 1999. A Epidemia de HIVIAIDS na Cidade do Rio de Janeiro e seus Padrões de Espacialidade. Dissertação de Mestrado, Rio de Janeiro: Escola Nacional de Saúde Pública, Fundação Oswaldo Cruz.

DINIZ, S. G. \& VILLELA, W. V., 1999. Interfaces entre os programas de DST/AIDS e saúde reprodutiva: O caso brasileiro. In: Saúde, Desenvolvimento e Política - Resposta Frente à AIDS no Brasil (R. Parker, J. Galvão \& M. Bessa, org.), pp. 123-176, Rio de Janeiro: Editora 34.

GOULD, P., 1993. The Slow Plague - A Geography of the AIDS Epidemic. Oxford: Blackwell.

GRANGEIRO, A., 1994. O perfil socioeconômico dos casos de AIDS na Cidade de São Paulo. In: A AIDS no Brasil (R. Parker, C. Bastos, J. Galvão \& J. S. Pedrosa, org.), pp. 91-125, Rio de Janeiro: RelumeDumará/Associação Brasileira Interdisciplinar de AIDS/Instituto de Medicina Social, Universidade do Estado do Rio de Janeiro.

IBGE (Fundação Instituto Brasileiro de Geografia e Estatística), 1991. Censo Demográfico, 1991. Rio de Janeiro: IBGE.
MS (Ministério da Saúde), 2000. Mulheres Aprovam Camisinha Feminina. 20 Fevereiro $2000<$ http:// www.aids.gov.br/cgi-bin/release.idc>.

PARKER, R., 1994. A Construção da Solidariedade AIDS, Sexualidade e Política no Brasil. Rio de Janeiro: Relume Dumará/Associação Interdisciplinar de AIDS/Instituto de Medicina Social, Universidade do Estado do Rio de Janeiro.

PARKER, R. \& CAMARGO Jr., K. R., 2000. Pobreza e HIV/AIDS: Aspectos antropológicos e sociológicos. Cadernos de Saúde Pública, 16(Sup. 1):89102

PARKER, R. G.; EASTON, D. \& KLEIN, C. H., 2000 Structural barriers and facilitators in HIV prevention: A review of international research. AIDS, 14 (Sup. 1):S22-S32.

SANTOS, S. M.; PINA, M. F. \& CARVALHO, M. S., 2000. Os sistemas de informação geográfica. In: Conceitos Básicos de Sistema de Informação Geográfica e Cartografia Aplicados à Saúde (M. F. Pina, M. S. Carvalho, \& S. M. Santos, org.), pp. 13-40, Brasília: Organização Pan-Americana da Saúde.

SZWARCWALD, C. \& BASTOS, F. I., 2000. AIDS e pauperização: Principais conceitos e evidências empíricas. In: Cadernos de Saúde Pública, 16(Sup. 1):65-76.

SZWARCWALD, C. L.; BASTOS, F. I.; BARCELLOS, C.; PINA, M. F. \& ESTEVES, M. A., 2000a. Health conditions and residential concentration of poverty: A study in Rio de Janeiro, Brazil. Journal of Epidemiology and Community Health, 54:530-536.

SZWARCWALD, C. L.; BASTOS, F. I.; ESTEVES, M. A. P. \& ANDRADE, C. L. T., 2000b. A disseminação da epidemia de AIDS no Brasil no período de 19871996: Uma análise espacial. Cadernos de Saúde Pública, 16(Sup. 1):7-19.

VERMELHO, L. L., 1999. Mulheres com AIDS: Desvendando histórias de risco. Cadernos de Saúde Pública, 15:369-379.

WALLACE, D. \& WALLACE, R., 1995. U.S. Apartheid and the spread of AIDS to the suburbs: A multicity analysis of the political economy of spatial epidemic threshold. Social Science and Medicine, 41:333-345.

WALLACE, R.; HUANG, Y.; GOULD, P. \& WALLACE, D. 1997. The hierarchical diffusion of AIDS and violent crime among US metropolitan regions: Innercity decay, stochastic resonance and reversal of the mortality transition. Social Science and Medicine, 44:935-947.

WALLACE, R. \& WALLACE, D., 1997. Socioeconomic determinants of health: Community marginalisation and the diffusion of disease and disorder in the United States. BMJ, 314:1341-1345.

Recebido em 3 de setembro de 2002

Versão final reapresentada em 19 de novembro de 2002 Aprovado em 14 de março de 2003 